\title{
New Creep Deformation Concept Based on Creep under Lower Stresses
}

Takashi Matsuo

\author{
Department of Metallurgy and Ceramics Science, Tokyo Institute of Technology, \\ 2-12-1, O-okayama, Meguro-ku, Tokyo 152-8552, Japan, tmatsuo@mtl.titech.ac.jp
}

Keywords: Single crystal, Schmidt Factor, Stress axis, Creep Deformation, Transient stage, Dynamic recrystalization.

Abstract. Through the analysis of many creep rate-time or creep rate-strain curves of $\gamma$-single phase Ni-20mass\% Cr alloy single crystals with various stress axes, it was clarified that the creep deformation manners at lower stresses are drastically different to those at higher stresses.

These creep features at lower stresses are summarized into three ones as follows.

(i) The fully extended transient stage occupies the considerable ratio of rupture life.

(ii) The steady state stage disappears, because the transient stage directly connected with the accelerating stage.

(iii) The origin of the onset of accelerating stage is regarded as the formation of the dynamic recrystallized grain.

These difference in creep deformation manner were caused by the predominant operation of the primary slip system and then the homogeneous evolution of dislocation substructures.

\section{Introduction}

Creep deformation well understood. The creep deformation manner defined as dislocation creep was composed mainly by J.Weertman [1] and a few researchers [2,3,4], and the most of their works were based on the creep curves of pure aluminum as shown in Fig. 1 [4]. Using this Figure, it was indicated that the creep strain in transient stage $\varepsilon_{\text {t }}$, and the strain during steady state creep stage $\varepsilon_{\mathrm{s}}$, are insensitive to temperature, but are slightly sensitive to applied stress. That is, the strains of $\varepsilon_{\mathrm{s}}$ become larger with increasing the stress. Here, it is notable that most of these creep tests had been carried out in a shorter time for less than $100 \mathrm{~h}$, in other words at higher stress. Under such conditions, the steady state stage has been regarded as the most important stage in creep, because it has been believed that the ratio of the transient stage to rupture life is quite smaller than the other two stages, and the accelerating stage is regarded as the dangerous stage.

In practical alloys, it has been also believed that the large part of the steady state stage turns to the accelerating stage [5], because of the coarsening of despersoids, as the result the ratio of accelerating stage to rupture life becomes larger. 
Based on this conception, the $\theta$ projection methods [6] and the $\omega$ method [7] were proposed as the methods to predict the creep curve and to estimate the creep life. The creep curves estimated by these two methods were composed only by the accelerating stage and the transient stage was completely ignored.

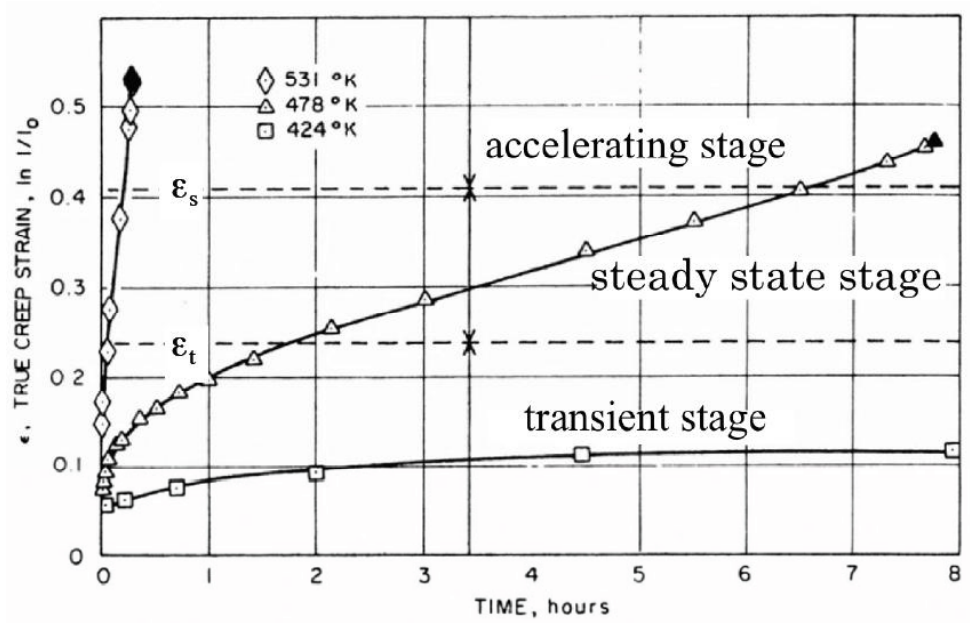

Fig. 1 Typical creep curves of high purity aluminum at three different temperatures under the stress of $20.6 \mathrm{MPa}$ [4].

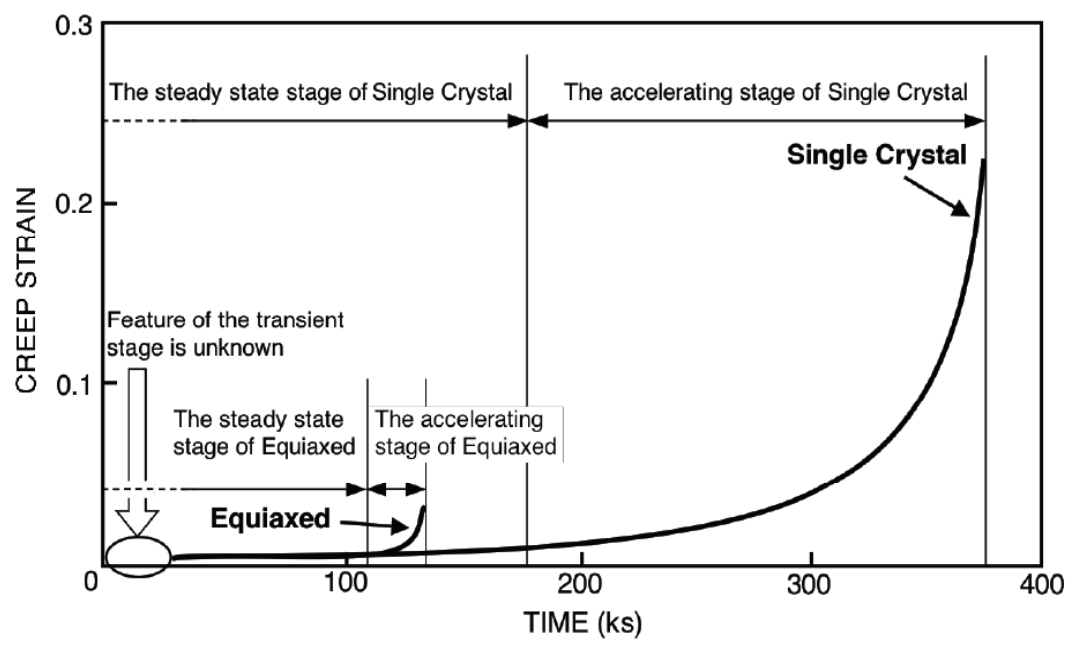

Fig. 2 Extension of the steady state stage and the accelerating stage by changing the specimen from conventional casted one to single crystal [8].

Intent to conduct the creep of $\gamma$ single phase single crystal. So far the origin of onset of accelerating creep has been regarded as the formation of voids and cracks on grain boundary. Therefore, the creep curve in single crystal removing the grain boundary has been characterized by the curve having the extended steady state and accelerating stages as shown in Fig. 2 [10]. To confirm these features, the creep tests at lower stresses using single crystals are conducted as follows.

\section{Experimental Procedure}

The $\gamma$ single phase $\mathrm{Ni}-20 \mathrm{Cr}$ single crystal with $13 \mathrm{~mm}$ in diameter and $280 \mathrm{~mm}$ in length was prepared 
in the modified Bridgeman type furnace. Three full sized creep specimens can be obtained from one single crystal. Stress axis of the single crystal was controlled by using the seed crystal. Creep tests were conducted at $1173 \mathrm{~K}$ under the stresses of 19.6 to $98.0 \mathrm{MPa}$. The lower stress is defined as the stress derived no instantaneous plastic strain, just after loading. After measuring the instantaneous strain for single crystals with different stress axis, the stress of $29.4 \mathrm{MPa}$ is defined as the lower stress for all the single crystals with various stress axes. Creep elongation was measured using DC type Differential Transformer through the extensometer attached on the edge portion of the full sized creep specimen with $6 \mathrm{~mm}$ in diameter of gage portion and $28 \mathrm{~mm}$ in length of gage portion. All the creep tests ware conducted using tensile creep testing machine under the constant stress.

\section{Result and discussion}

Transient stage in single crystal under the lower stress. Firstly, creep rate-time curves at $1173 \mathrm{~K}$ in the stress range of 19.6 to $98.0 \mathrm{MPa}$, using the single crystals with stress axes within the standard stereographic triangle are shown in Fig. 3. In every curves, the stage where the creep rate remains constant appears just after loading, and this stage connects to the stage showing the steep decrease in creep rate. The former stage and latter stage are designated as PSO stage and DSE stage, respectively. PSO stage indicates the Primary Slip system Operating stage, and DSE stage indicates the Dislocation Substructure Evolving stage. It is noteworthy that DSE stage is followed not by the steady stage but by the accelerating stage. Though the decrease in creep rate in DSE stage becomes steeper in the curves at the stress below $49 \mathrm{MPa}$, the appearance of PSO stage must be regarded as the most characterized features of single crystal. Here, it is presumed that the deformation in PSO stage proceed by the operation of primary slip system of $[, 101](111)$, and further operation of the secondary and third slip systems lead to DSE stage. Therefore, PSO stage and DSE stage could be regarded as the transient stage in creep well understood. It is notable that these deformation manners are completely different from those well understood. The proceeding of creep deformation at lower stress is considered as follows, firstly the deformation through the primarily slip system proceeds, then the stress axis moves for [001]-[,111] line, after arriving at the area near [001]-[,111] line, secondary and third slip systems operate, and finally the dynamic recrystalization occurs, and the extending of the dynamic recrystalization leads to rupture. By supposing these deformations, there are two important points, first one is the fact that the moving of stress axes makes the creep rate small through the decreasing in Schmid Factor, and second one is the fact that operations of slip systems proceed gradually and uniformly. First one indicates the continuation of transient stage, and second one indicates the impossibility of the early appearance of steady state. These deformation manner cannot be applied to the single crystals with stress axis of three pole positions of [001], [,111] and [011] and in the area which the orientation of stress axis to slip plane is more than $45^{\circ}$. But in these single crystals, transient stage occupies the larger ratio of rupture life. 


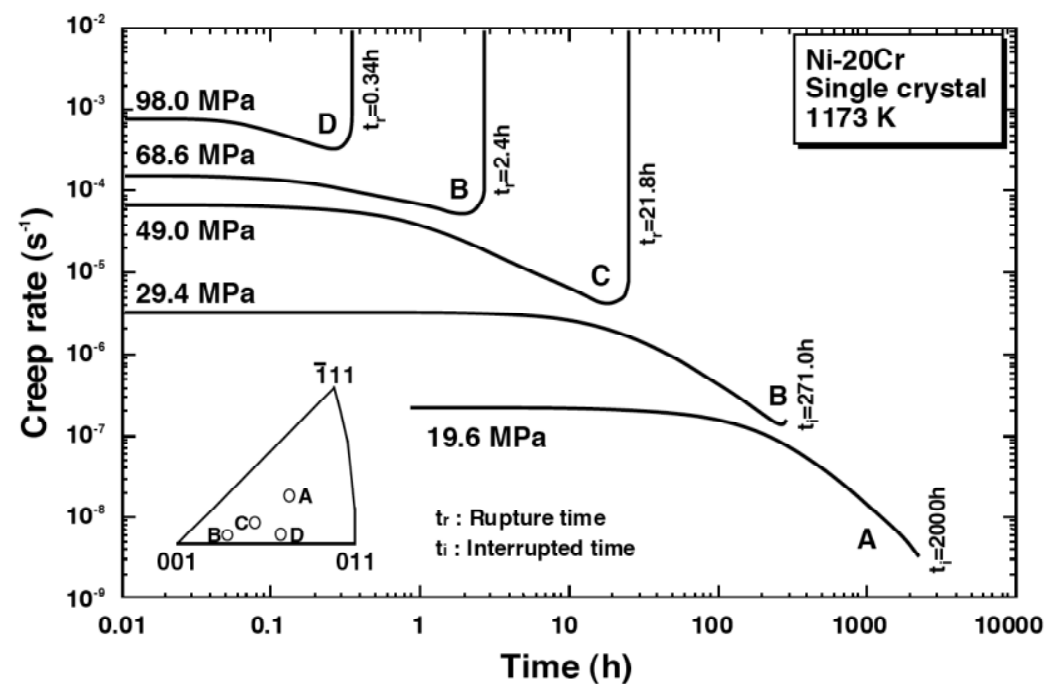

Fig. 3 Creep rate-time curves in the stress range of 19.6 to $98.0 \mathrm{MPa}$ at $1173 \mathrm{~K}$ using single crystals with stress axes within the standard stereographic triangle.

Origin of onset of accelerating creep. It has been believed that the onset of accelerating creep was caused by the formation of cracks and voids. The creep interrupting tests were conducted at the strains of $0.10,0.50,0.73,0.76$ and 0.83 as shown in Fig. 4, Optical microstructural observations were done for all interrupted specimens. As the results it was confirmed that the onset of accelerating stage is quite corresponded to the appearance of dynamic recrystalization through the evolution of subgrains as shown in Fig. 5. The formations of voids and cracks were confirmed on the grain boundary at the end of accelerating creep stage.

But it was not well understood that the dynamic recrystalization leads onset of accelerating creep, because the creep rate was not depend on grain size in dislocation creep. To interpret the grain size dependence of creep rate in dislocation creep, the Core Mantle Model was proposed by Terada et al. [11]. According to this model, the portion along grain boundary where dislocation substructures is free increases with decreasing the grain size. And this portion along grain boundary was designated as Mantle. By using this model, the increase in creep rate during the accelerating stage can be interpreted.

Application of deformation manner in single crystal to polycrystal materials. According to these creep deformation manners in single crystal, two suppositions of the creep deformation manners in polycrystal were given. First one is the appearance of PSO stage just after loading. And second one is the moving of the stress axes for [001]-[,111] line in standard triangle of each crystals composed polycrystal. To clarify these two suppositions, the creep test at 29.4MPa using Ni-20Cr polycrystal with grain diameter of $1060 \mu \mathrm{m}$ was interrupted at the beginning of the accelerating stage. Just after loading, PSO stage was detected. The inverse pole figures of the solution treated specimen and the creep interrupted specimen are shown in Fig. 6. The spots of the stress axes of each grains of solution treated specimen were dispersed in the whole region, while the spots of the stress axes of the 


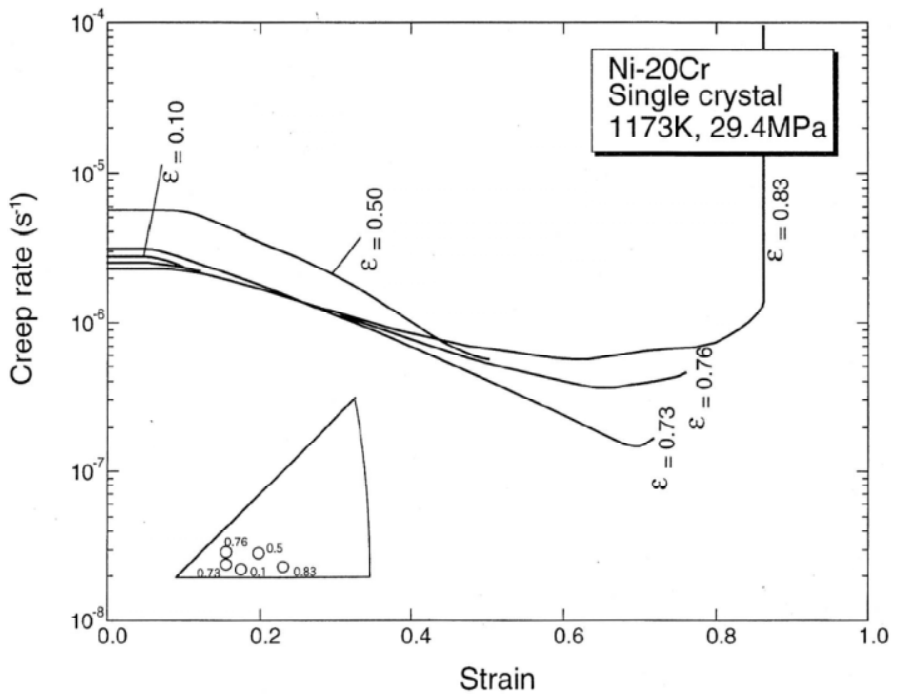

Fig. 4 Five creep rate-strain curves at 29.4MPa, creep interrupted at the strains of 0.10,

$0.50,0.73,0.76$ and 0.80 . Their stress axes are shown in standard steleographic triangle.
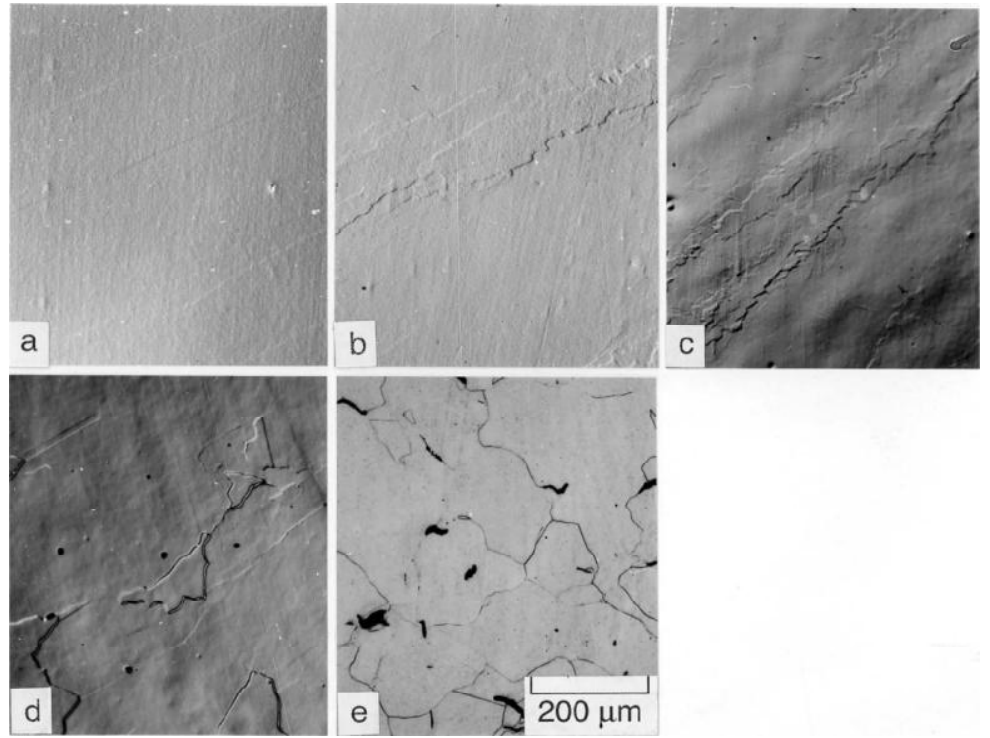

Fig. 5 Optical micrographs of the specimens creep interrupt at the strains of $0.10(a), 0.50(b), 0.73(c)$,

0.76(d), and 0.83(e), showing the dynamic recrystalized grains through evolution of subgrains.

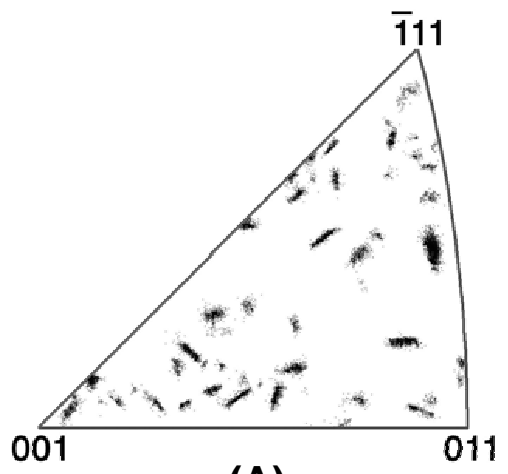

(A)

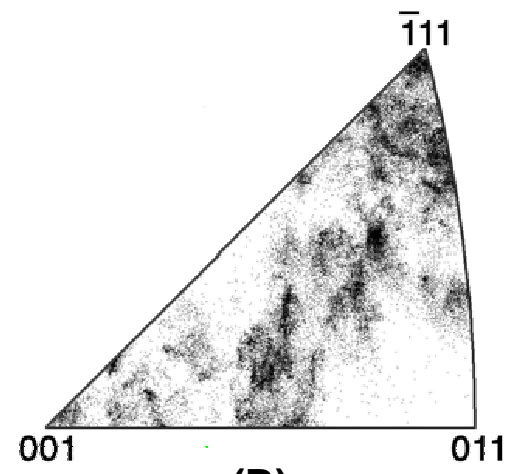

(B)

Fig. 6 Inverse pole figures of solution treated Ni-20Cr polycrystal with grain diameter of $1060 \mu \mathrm{m}(\mathrm{A})$, and that of creep interrupted specimen at the beginning of accelerating stage (B). 
grains in the specimen creep interrupted move for the region near [001]-[,111] line, especially many spots gathered at the area near the pole of [,111].

\section{Conclusions}

By investigating the creep rate-time curves or creep rate-strain curves of $\gamma$ single phase $\mathrm{Ni}-20 \mathrm{Cr}$ single crystals conducted the creep tests at lower stress at $1173 \mathrm{~K}$, it was clarified that the creep deformation manners at lower stresses are drastically different to those at higher stresses.

These creep features at lower stresses are summarized into three ones as follows.

(i) The fully extended transient stage occupies the considerable ratio of rupture life.

(ii) The steady state stage disappears, because the transient stage directly connected with the accelerating stage.

(iii) The origin of the onset of accelerating stage is regarded as the formation of the dynamic recrystallized grain.

These difference in creep deformation manner were caused by the predominant operation of the primary slip system and then the homogeneous evolution of dislocation substructures.

\section{References}

[1] J. Weertman, Trans. ASM, 61 (1968), p. 681.

[2] A. K. Mukherjee, J. E. Bird and J. E. Dorn, Trans. ASM, 62 (1969), p. 155

[3] J. E. Bird, A.K. Mukherjee and J. E. Dorn, Quantitative Relation between Properties and Microstructure, Israel Universities Press, Haba, Israel, (1969), p. 255.

[4] H. I. Lial Huang, O. D. Sherby and J. E. Dorn, Trans. AIME, 206 (1956), p. 1385.

[5] H. Ota, K. Maruyama and H. Oikawa, "Strength of Metals and Alloys", Ed. D. G. Brandon et al, Freund Publ. House, London, (1991), p. 343

[6] R. W. Evans, and B. Wilshire, Creep of Metals and Alloys, The Institute Metals, London, (1985), p. 197.

[7] B. J. Cane, Mater. Forum, 9 (1986), p. 5.

[8] K. Kimura, T. Kisanuki, S. Komatsu, T. Matsuo and R. Tanaka, Tetsu-To-Hagane, 71 (1985), p. 1803

[9] Final Report of Research Group on Strengthening of Heat Resisting Steel and Alloy, JISI, (2000), p. 177

[10] F. L. Ver Snyder and M. E. Shank, Mats. Sc. Engrg, 6 (1970), p. 213

[11] Y. Terada, T. Matsuo and M. Kikuchi, Aspect of High Temperature Deformation and Fracture, Ed. By Hosoi et al., Japan Institute of metal, (1993), p. 27 